\title{
Long-term outcome following concomitant mitral valve surgery and Cox maze procedure for atrial fibrillation
}

\author{
Niv Ad, MD, ${ }^{\mathrm{a}, \mathrm{b}, \mathrm{c}}$ Sari D. Holmes, PhD, ${ }^{\mathrm{d}}$ Paul S. Massimiano, MD, ${ }^{\mathrm{b}}$ Anthony J. Rongione, MD, ${ }^{\mathrm{b}}$ and \\ Lisa M. Fornaresio, $\mathrm{PhD}^{\mathrm{d}}$
}

\section{ABSTRACT}

Objective: Atrial fibrillation (AF) is associated with increased early and longterm morbidity/mortality following valve surgery. This study examined longterm influence of concomitant full Cox maze (CM) and mitral valve procedures on freedom from atrial arrhythmia and stroke.

Methods: This sample comprised patients who underwent CM with a mitral valve procedure $(\mathrm{N}=473)$. Data on rhythm, medication status, and clinical events captured according to Heart Rhythm Society guidelines at 6, 9, 12, 18, and 24 months and yearly thereafter up to 7 years.

Results: Mean age was 65 years, mean left atrium size was $5.3 \mathrm{~cm}$, and $15 \%$ had paroxysmal AF. Perioperative stroke occurred in 2 patients $(0.4 \%)$ and operative mortality was $2.7 \%(n=13)$. Return to sinus rhythm regardless of antiarrhythmic drugs at 1,5 , and 7 years was $90 \%, 80 \%$, and $66 \%$. Sinus rhythm off antiarrhythmic drugs at 1,5 , and 7 years was $83 \%, 69 \%$, and $55 \%$. Freedom from embolic stroke at 7 years was $96.6 \%$ (0.4 strokes per 100 patient-years) with a majority of patients off anticoagulation medication. Greater odds of atrial arrhythmia recurrence during 7 years was associated with longer AF duration (odds ratio $[\mathrm{OR}], 1.07 ; P=.001$ ), whereas lower odds were associated with cryothermal energy only (OR, $0.64 ; P=.045)$ and greater surgeon experience (OR, $0.98 ; P=.025)$.

Conclusions: This study suggests that the addition of CM to mitral valve procedures, even with a high degree of complexity, did not increase operative risk. In long-term follow-up, the CM procedure demonstrated acceptable rhythm success, reduced AF burden, and remarkably low stroke rate. Individual surgeon experience and training may notably influence long-term surgical ablation for AF success. (J Thorac Cardiovasc Surg 2018;155:983-94)

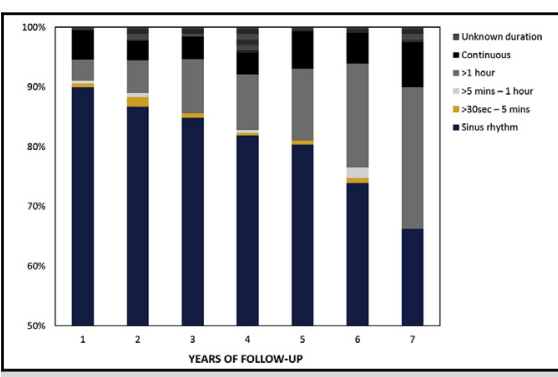

Burden of atrial arrhythmias during 7 years of followup.

\section{Central Message}

In long-term follow-up, the Cox maze procedure demonstrated acceptable rhythm success, reduced atrial fibrillation burden, and low stroke rate. This evidence should support decision making for surgical ablation for atrial fibrillation.

\section{Perspective}

Surgical ablation for atrial fibrillation remains underperformed despite ablation device improvements and growing evidence on its positive influence. One significant gap in the literature is lack of substantial data regarding long-term success and clinical influence of surgical ablation for atrial fibrillation. This study demonstrated that patients undergoing mitral valve surgery with Cox maze procedures should expect good early and late outcomes.

See Editorial Commentary page 995

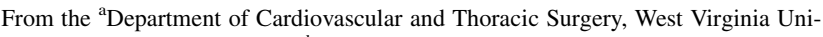
versity, Morgantown, WVa; ${ }^{b}$ Washington Adventist Hospital, Takoma Park, Md; ${ }^{\mathrm{c}}$ Inova Fairfax Hospital, Falls Church, Va; and ${ }^{\mathrm{d}}$ West Virginia University Heart and Vascular Institute, West Virginia University, Morgantown, WVa.

Read at the 97th Annual Meeting of The American Association for Thoracic Surgery, Boston, Massachusetts, April 29-May 3, 2017.

Received for publication May 17, 2017; revisions received Aug 31, 2017; accepted for publication Sept 21, 2017; available ahead of print Dec 13, 2017.

Address for reprints: Niv Ad, MD, Department of Cardiovascular and Thoracic Surgery, West Virginia University, 1 Medical Center Dr, Morgantown, WV 26506 (E-mail: nivadmd14@gmail.com).

$0022-5223 / \$ 36.00$

Copyright $(2017$ by The American Association for Thoracic Surgery

https://doi.org/10.1016/j.jtcvs.2017.09.147
}

Atrial fibrillation (AF) is associated with increased morbidity, decreased survival, and inferior quality of life. ${ }^{1-3}$ Despite the significant improvement in surgical ablation devices and growing evidence regarding the positive influence of surgical ablation on patient outcomes, surgical ablation for AF is still underperformed

Scanning this QR code will take you to the article title page. To view the AATS Annual Meeting Webcast, see the URL next to the webcast thumbnail.

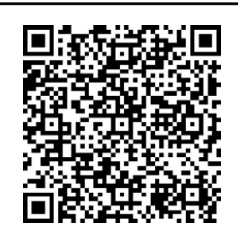




\section{Abbreviations and Acronyms \\ $\mathrm{AAD}=$ antiarrhythmic drugs \\ $\mathrm{AF}=$ atrial fibrillation \\ $\mathrm{CM}=$ Cox maze procedure \\ HSR $=$ Heart Rhythm Society}

and many patients are left untreated. ${ }^{4}$ Recent guidelines by surgical organizations support the performance of concomitant surgical ablation for AF when indicated. These guidelines clearly state that surgical ablation is effective and safe. ${ }^{5,6}$ It is especially important to notice that surgical ablation was found to be associated with improved perioperative and late survival and reduced risk of strokes in long-term follow-up. ${ }^{6}$ It is therefore correct to assume that if $\mathrm{AF}$ is left untreated it may be associated with increased long-term mortality and morbidity in patients undergoing valve surgery. ${ }^{7,8}$ Among the most significant gaps in the current literature is the lack of substantial data regarding the long-term success and potential clinical influence of surgical ablation for AF on outcome. The purpose of this study was to examine the long-term influence of the concomitant full Cox maze (CM) procedure and mitral valve surgery on freedom from atrial arrhythmia and stroke, and to identify the variables associated with long-term success of the CM procedure.

\section{METHODS}

Patients who underwent concomitant $\mathrm{CM}$ procedures at a single center since September 2005 were part of a prospective follow-up program $(\mathrm{N}=714)$ of which 473 patients had a mitral valve procedure. Data on rhythm, medication status, follow-up interventions, and clinical events were captured according to the Heart Rhythm Society (HRS) guidelines at 6, 9, 12, 18, and 24 months and yearly thereafter up to 7 years. Data on survival and clinical events during follow-up were collected throughout the entirety of follow-up. Therefore, data were available for these outcomes even when rhythm status was unavailable. Sinus rhythm status was defined according to HRS guidelines as freedom from atrial arrhythmia $>30$ seconds without use of class I/III antiarrhythmic drugs (AADs). ${ }^{9}$ This study was approved by an institutional review board and written patient consent was waived.

\section{Surgical Procedure}

The CM III/IV procedures were performed by multiple surgeons and the lesion set was applied as previously described. ${ }^{10,11}$ The energy source used for each procedure was based on surgeon discretion. The lesion set for the cryothermal-only procedures was the traditional CM III procedure and for the cryothermal combined with bipolar radiofrequency energy procedures was the CM IV lesion set. ${ }^{10-12}$ Bipolar radiofrequency was applied 3 to 5 times across the pulmonary veins (Isolator Synergy Clamps; AtriCure Inc, Mason, Ohio). For cryothermal energy only the CryoFlex (10 or 10S; Medtronic, Minneapolis, Minn) or the Cryo 1 Cryo 2 (AtriCure Inc) were used. For cryothermal energy only or in combination with bipolar radiofrequency, the cryolesions were applied for 2 minutes for all lesions crossing the atrioventricular groove both in the right and left atria and the epicardial coronary sinus lesion to complete the left atrial isthmus lesion on the left. The left atrial appendage was managed either by amputation and suture closure or by applying an epicardial clip (AtriCure Inc). In redo cases and minimally invasive right thoracotomy, the left atrial appendage was sutured closed endocardially in 2 layers. Appropriate left atrial appendage closure was confirmed in all cases via transesophageal echocardiography in the operating room.

\section{Patient Management}

The management of patients in regard to medications and follow-up interventions after surgery was determined by an institutional protocol. ${ }^{13}$ Unless contraindicated, all patients received amiodarone at $200 \mathrm{mg}$ twice a day for 5 days after surgery followed by $200 \mathrm{mg}$ daily for 3 months to reduce the risk of early atrial arrhythmia recurrence. ${ }^{14}$ If patients were in stable sinus rhythm at 3 months after surgery, amiodarone was recommended to be discontinued to allow for at least 6 weeks off the medication by the 6 months follow-up time point. Patients with an amiodarone allergy, history of side effects due to amiodarone, or other clinical contraindications were generally treated with sotalol or flecainide instead, which was prescribed for 3 months after surgery according to the same protocol.

Similarly, unless contraindicated due to high risk for bleeding, all patients were treated with warfarin to maintain international normalized ratio between 2 and 2.5 or higher in applicable cases (eg, mechanical valve implantation). If patients were in stable sinus rhythm off AADs at 6 months after surgery and echocardiography demonstrated a wellexcluded or excised left atrial appendage with no signs of blood stagnation in the left heart cavities (ie, smoke), discontinuation of anticoagulation was recommended and patients were maintained on aspirin. Discontinuation of anticoagulation was considered throughout followup if patients were qualified, but was under the direction of each individual cardiologist.

\section{Statistical Analysis}

All analyses were conducted using IBM SPSS Statistics version 24.0 (IBM Corp, Armonk, NY) and a $P$ value of $<.05$ two-tailed was considered statistically significant. Continuous data are presented as mean \pm standard deviation or median (interquartile range [IQR]) and categorical data are presented as frequency (\%). Descriptive statistics were used to illustrate the characteristics and outcomes of the sample $(\mathrm{N}=473)$. Missing values in patient characteristics were present in preoperative left atrium size $(n=22)$ and duration of $\mathrm{AF}(\mathrm{n}=33)$. Multiple imputation was conducted to provide complete data on these 2 variables using left atrium size, duration of $\mathrm{AF}$, and age in the imputation model with 3 iterations. The mean of the 3 iterations was calculated and used to replace the missing values for left atrium size and duration of AF. Patients with mitral valve as the only concomitant procedure and those with mitral and other concomitant procedures were compared on preoperative characteristics and rhythm outcomes using independentsamples $t$ tests or Mann-Whitney $U$ tests for continuous variables and $\chi^{2}$ or Fisher exact test for categorical variables, as appropriate.

Kaplan-Meier survival analysis was conducted to examine freedom from events and mortality during follow-up. A generalized linear mixed model using multivariable binomial logistic regression was conducted to examine the risk factors for recurrence of atrial arrhythmia up to 7 years after surgery. Not all patients in this sample had reached 7 years after surgery, but the mixed model methodology with random intercepts allowed the inclusion of all available follow-up rhythm data and individualspecific random effects. The fixed effects factors included in this model were selected a priori based on clinical and theoretical justification and included age, gender, ejection fraction, left atrium size $(\mathrm{cm})$, duration of $\mathrm{AF}$ (years), type of $\mathrm{AF}$ as defined by the HRS guidelines (paroxysmal AF vs nonparoxysmal AF), energy source (cryothermia alone vs all others), operative approach (minimally invasive vs median sternotomy), consecutive cases of surgeon experience with surgical ablation at the time of the procedure (continuous variable in 20 case intervals), and mitral valve procedure (repair vs replacement). 
TABLE 1. Patient characteristics for the total sample and by type of concomitant procedures

\begin{tabular}{|c|c|c|c|}
\hline Characteristic & Total sample $(N=473)$ & Mitral valve only $(n=217)$ & Mitral valve + others $(n=256)$ \\
\hline $\operatorname{Age}(y)^{*}$ & $65.3 \pm 11.4$ & $63.9 \pm 11.3$ & $66.4 \pm 11.4$ \\
\hline Female & $212(45)$ & $102(47)$ & $110(43)$ \\
\hline Body mass index & $27.1 \pm 5.8$ & $27.0 \pm 5.7$ & $27.3 \pm 6.0$ \\
\hline Ejection fraction $(\%) *$ & $54.6 \pm 11.0$ & $56.2 \pm 10.1$ & $53.3 \pm 11.5$ \\
\hline Diabetes & $49(10)$ & $16(7)$ & $33(13)$ \\
\hline Hypertension* & $289(61)$ & $122(56)$ & $167(65)$ \\
\hline Peripheral vascular disease & $27(6)$ & $11(5)$ & $16(6)$ \\
\hline Previous cerebrovascular accident & $26(6)$ & $8(4)$ & $18(7)$ \\
\hline Chronic pulmonary disease & $112(24)$ & $46(21)$ & $66(26)$ \\
\hline Duration of AF (y) & $1.5(0.2-4.7)$ & $1.1(0.2-4.4)$ & $1.7(0.3-5.1)$ \\
\hline Left atrium size $(\mathrm{cm})$ & $5.3 \pm 1.0$ & $5.3 \pm 1.0$ & $5.3 \pm 1.0$ \\
\hline Nonparoxysmal AF type* & $405(86)$ & $176(81)$ & $229(90)$ \\
\hline $\mathrm{CHADS}_{2}$ score $\geq 2 *$ & $210(44)$ & $78(36)$ & $132(52)$ \\
\hline EuroSCORE II $(\%)^{*}$ & $5.0 \pm 5.4$ & $3.3 \pm 3.5$ & $6.4 \pm 6.3$ \\
\hline Elective status* & $400(85)$ & $193(89)$ & $207(81)$ \\
\hline Minimally invasive* & $52(11)$ & $48(22)$ & $4(2)$ \\
\hline Cryothermia only energy source & $258(55)$ & $126(58)$ & $132(52)$ \\
\hline Cases of surgeon experience & $218(70.5-480.5)$ & $230(83-482.5)$ & $211(54-476)$ \\
\hline \multicolumn{4}{|l|}{ Concomitant procedures $\dagger$} \\
\hline CABG surgery & $82(17)$ & - & $82(32)$ \\
\hline Aortic valve surgery & $47(10)$ & - & 47 (18) \\
\hline Tricuspid valve surgery & $144(30)$ & - & $144(56)$ \\
\hline
\end{tabular}

Values are presented as frequency ( $\%)$, mean \pm standard deviation, or median (interquartile range). AF, Atrial fibrillation; EuroSCORE, European System for Cardiac Operative Risk Evaluation; $C A B G$, coronary artery bypass grafting. *Comparison of mitral valve only and mitral valve + others $(P<.05)$. $\dagger$ Categories are not mutually exclusive.

\section{RESULTS}

The mean age for this sample was $65.3 \pm 11.4$ years, mean European System for Cardiac Operative Risk Evaluation (EuroSCORE) II was $5 \% \pm 5.4 \%$, and $45 \%$ were female patients (Table 1). The mean left atrium size was $5.3 \pm 1.0 \mathrm{~cm}$ with left atrium size $>5.5 \mathrm{~cm}$ in $37 \%$ of patients, median preoperative duration of $\mathrm{AF}$ was 1.5 years (IQR, 0.2-4.7 years), and $15 \%$ of patients with paroxysmal $\mathrm{AF}$ type. Preoperative duration of $\mathrm{AF}$ was longer than 3 years in $36 \%$ and longer than 5 years in $24 \%$ of patients. In addition, $9 \%$ of patients had left atrium size $>5.5 \mathrm{~cm}$ and $\mathrm{AF}$ duration $>5$ years. Mitral valve was the only concomitant procedure in $46 \%$ of patients, $44 \%$ had 3 total procedures, and $10 \%$ had $4+$ procedures. Follow-up rhythm data were available on $92 \%$ of eligible patients at 1 year after surgery, $87 \%$ of eligible patients at 3 years after surgery, $80 \%$ of eligible patients at 5 years after surgery, and $75 \%$ of eligible patients at 7 years after surgery. Patients who withdrew or were lost to follow-up did not differ on patient characteristics from the patients with rhythm status available at 7 years after surgery.

\section{Rhythm Outcomes}

Return to sinus rhythm regardless of AADs at 1,5 , and 7 years was $90 \%, 80 \%$, and $66 \%$, respectively. Sinus rhythm off AADs at 1, 5, and 7 years was $83 \%, 69 \%$, and $55 \%$, respectively (Figure 1). At 7 years, monitoring revealed that only $22 \%$ of patients with recurrent atrial arrhythmia demonstrated a continuous pattern (Figure 2). The CM procedures in this series were performed by 8 separate surgeons and notable differences in success at 7 years were identified. The more experienced surgeons demonstrated better success than the rest with $50 \%$ to $73 \%$ at 7 years. Only 1 surgeon had more than 50 patients with at least 7 years of follow-up.

In the group of patients who underwent only mitral valve and CM procedures $(n=217)$, sinus rhythm regardless of AADs at 1,5 , and 7 years was $92 \%$ (153 out of 167 ), $86 \%$ (68 out of 79 ), and $80 \%$ (36 out of 45 ) and sinus rhythm off AADs at 1,5 , and 7 years was $85 \%$ (140 out of 165 ), $79 \%$ (62 out of 79 ), and $64 \%$ (29 out of 45 ). In contrast, the group of patients who underwent more than mitral valve and CM procedures, had fewer patients in sinus 


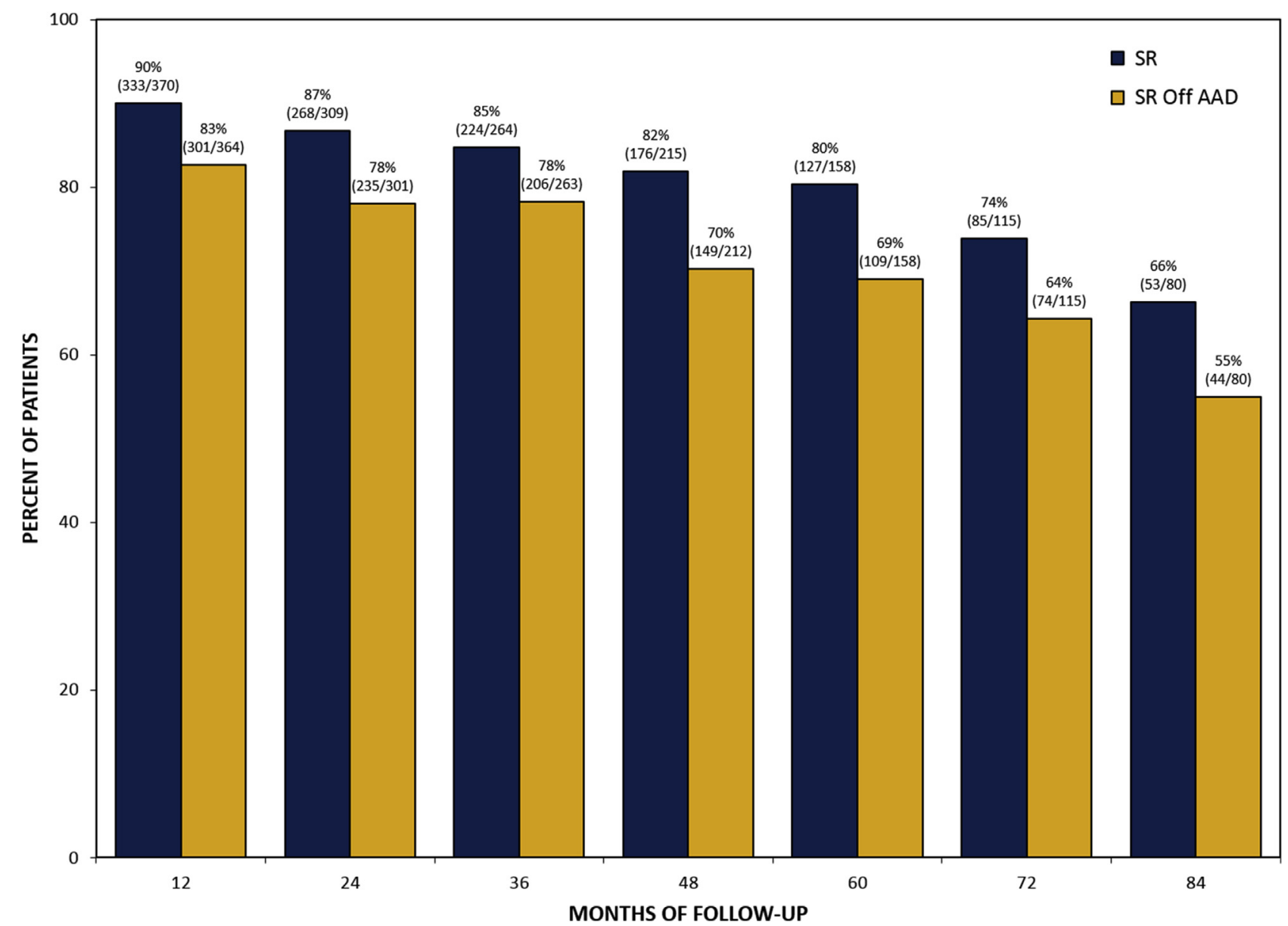

FIGURE 1. Percent of patients in sinus rhythm $(S R)$ and SR off antiarrhythmic drugs $(A A D)$ during 7 years of follow-up.

rhythm and sinus rhythm off AADs throughout follow-up. These comparisons reached significance for sinus rhythm regardless of AADs at 3 years $(80 \%$ vs $90 \% ; P=.035)$, 6 years $(62 \%$ vs $86 \% ; P=.004)$, and 7 years $(49 \%$ vs $80 \% ; P=.003)$ and for sinus rhythm off AADs at 3 years $(72 \%$ vs $85 \% ; P=.008), 5$ years $(60 \%$ vs $79 \% ; P=.010)$, and 6 years $(53 \%$ vs $75 \% ; P=.014)$. In the group of patients with mitral valve repair, return to sinus rhythm regardless of AADs at 1,5 , and 7 years was $92 \%, 82 \%$, and $73 \%$ and sinus rhythm off AADs was $86 \%, 70 \%$, and $58 \%$, respectively.

Mixed-model logistic regression analysis revealed that greater odds of atrial arrhythmia recurrence during 7 years of follow-up was associated with longer preoperative duration of AF in years $(P=.001)$, whereas lower odds of atrial arrhythmia recurrence was associated with cryothermal energy only $(P=.045)$ and greater cases of surgeon experience $(P=.025)$ (Table 2$)$. For each year increase in duration of $\mathrm{AF}$, there was a $7 \%$ greater risk for atrial arrhythmia recurrence during 7 years of follow-up and for each additional 20 cases of surgeon experience, there was a $2 \%$ reduction in risk for atrial arrhythmia recurrence. Other factors in the model were not significantly associated with atrial recurrence during 7 years, including age, mitral valve repair, nonparoxysmal AF type, and larger left atrial size (Table 2). The distribution lines presented in Figure 3 highlight the importance of AF duration rather than left atrial size in distinguishing patients in sinus rhythm off AADs at long-term follow-up.

\section{Clinical Outcomes}

The incidence of perioperative complications was low for these patients and included the following: pneumonia in $3 \%(\mathrm{n}=15)$, perioperative stroke in $0.4 \%(\mathrm{n}=2)$, reoperation for bleeding in $2.6 \%(\mathrm{n}=12)$, renal failure in $3 \%$ $(\mathrm{n}=16)$, renal failure requiring temporary dialysis in $2 \%$ $(\mathrm{n}=11)$, and readmissions $<30$ days in $11 \%(\mathrm{n}=52)$. New pacemakers were implanted in 37 of the 427 patients without a preoperative pacemaker $(9 \%)$. The indications for pacemaker implantation were sick sinus syndrome in 20 patients $(54 \%)$, complete atrioventricular block in $10 \mathrm{pa}-$ tients $(27 \%)$, bradycardia in 4 patients $(11 \%)$, and sinus 


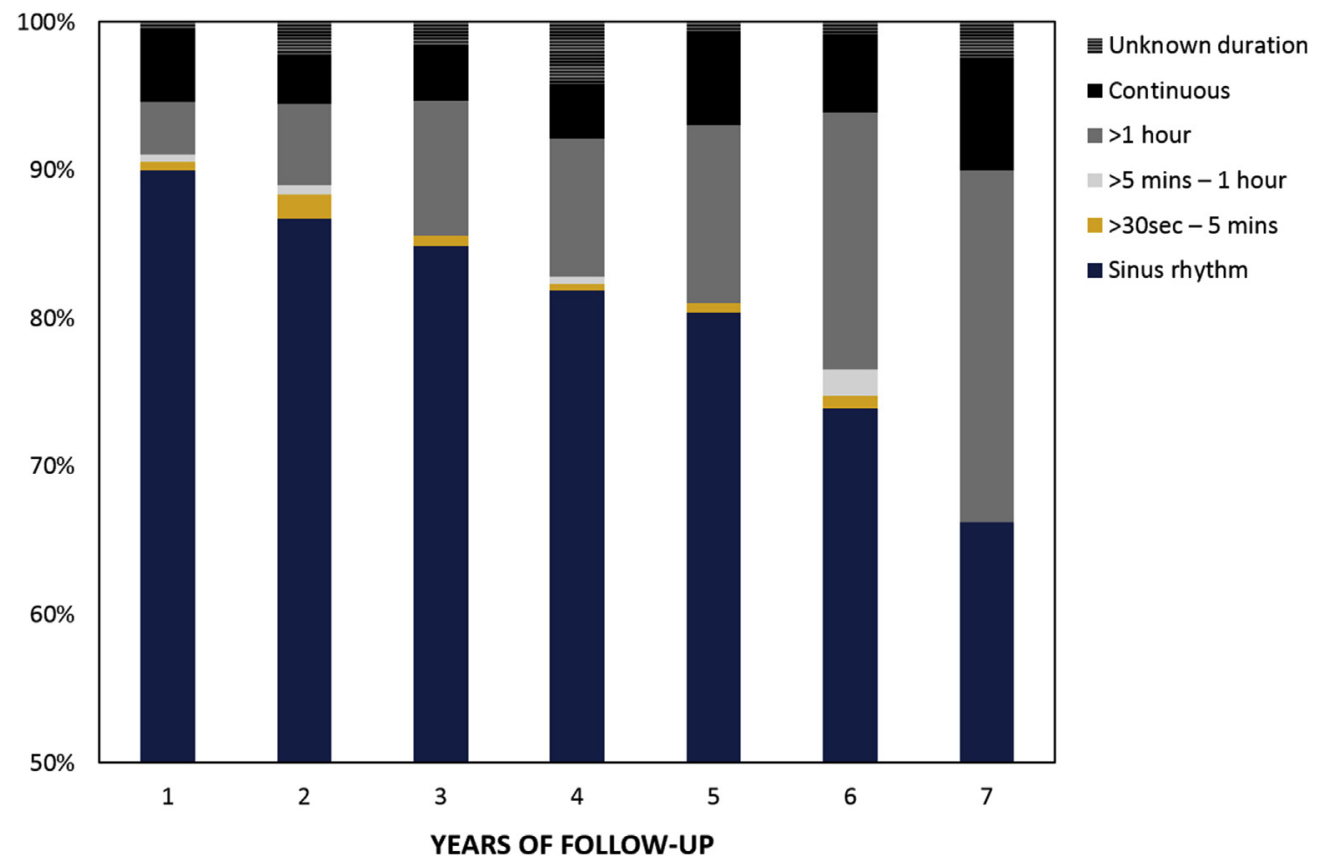

FIGURE 2. Burden of atrial arrhythmias during 7 years of follow-up.

node dysfunction in 3 patients $(8 \%)$. Intraoperative blood products were transfused in $24 \%$ of patients $(n=115)$ and blood products were transfused postoperatively in $32 \%(n=149)$. Specifically, red blood cell units were transfused intraoperatively in $11 \%$ of patients $(n=53)$ and red blood cell units were transfused postoperatively in $25 \%$ $(\mathrm{n}=120)$. Median intensive care unit length of stay was 45 hours (IQR, 12-95 hours) and median hospital length of stay was 7 days (IQR, 5-10 days). Operative

TABLE 2. Results of the mixed model logistic regression analysis for atrial arrhythmia recurrence

\begin{tabular}{lccc}
\hline \multicolumn{1}{c}{ Factor } & Odds ratio & $\begin{array}{c}\mathbf{9 5} \% \text { Confidence } \\
\text { interval }\end{array}$ & $\begin{array}{c}\boldsymbol{P} \\
\text { value }\end{array}$ \\
\hline Age (y) & 1.01 & $0.99-1.03$ & .208 \\
Female & 1.07 & $0.69-1.66$ & .773 \\
\hline Ejection fraction (\%) & 0.99 & $0.97-1.01$ & .430 \\
Left atrium size (cm) & 1.06 & $0.85-1.32$ & .614 \\
\hline Duration of AF (y) & 1.07 & $1.03-1.11$ & .001 \\
\hline Nonparoxysmal AF type & 1.05 & $0.57-1.94$ & .873 \\
\hline Surgeon experience & 0.98 & $0.96-0.997$ & .025 \\
Cryothermia only energy & 0.64 & $0.41-0.99$ & .045 \\
$\quad$ source & & & \\
\hline Minimally invasive & 0.99 & $0.47-2.06$ & .973 \\
Mitral valve repair & 0.68 & $0.43-1.06$ & .087 \\
\hline$A F$, Atrial fibrillation. & & &
\end{tabular}

The Journal of Thoracic and Cardiovascular Surgery • Volume 155, Number 3 mortality $<30$ days was $2.7 \%(\mathrm{n}=13)$ and the observedto-expected ratio was 0.55 using the EuroSCORE II as the expected value $(5 \%)$. At discharge, $88 \%$ of patients were in sinus rhythm (405 out of 462 patients), $66 \%$ were discharged on AADs (314 out of 462 patients), and 92\% were discharged on anticoagulation (425 out of 462 patients).

Mean follow-up was $52 \pm 37$ months (median, 48 months [IQR, 19-77 months]). Preoperative $\mathrm{CHADS}_{2}$ score was $\geq 2$ in $44 \%$ of patients and the median $\mathrm{CHADS}_{2}$ score was 1 (IQR, 1-2). Major bleeding events occurred in 44 patients $(9 \%)$ by 7 years and embolic strokes occurred in 9 patients $(2 \%)$ by 7 years. For patients with embolic strokes, median $\mathrm{CHADS}_{2}$ score was 2 (IQR, 0.5-2.5), mean left atrium size was $5.2 \pm 0.8 \mathrm{~cm}$, median preoperative $\mathrm{AF}$ duration was 2.3 years, 4 patients were taking anticoagulation medication at the time of the event for clinical indications, and $5 \mathrm{pa}-$ tients were in sinus rhythm. Of 4 patients not in sinus rhythm at the time of the embolic stroke, 3 were taking anticoagulation medication and 1 had unknown anticoagulation status. Cumulative freedom from embolic stroke at 7 years was $96.6 \%$ (95\% confidence interval, 0.941-0.992; 0.4 strokes per 100 patient-years of follow-up) (Figure 4). At 7 years, the majority of patients who could clinically discontinue anticoagulation had done so (41 out of 46 patients; $89 \%$ ) and $87 \%$ of patients were off AADs at 7 years. Although only $29 \%$ of patients remained on oral anticoagulation medication throughout follow-up, the incidence of embolic stroke was very low. Cumulative survival from 

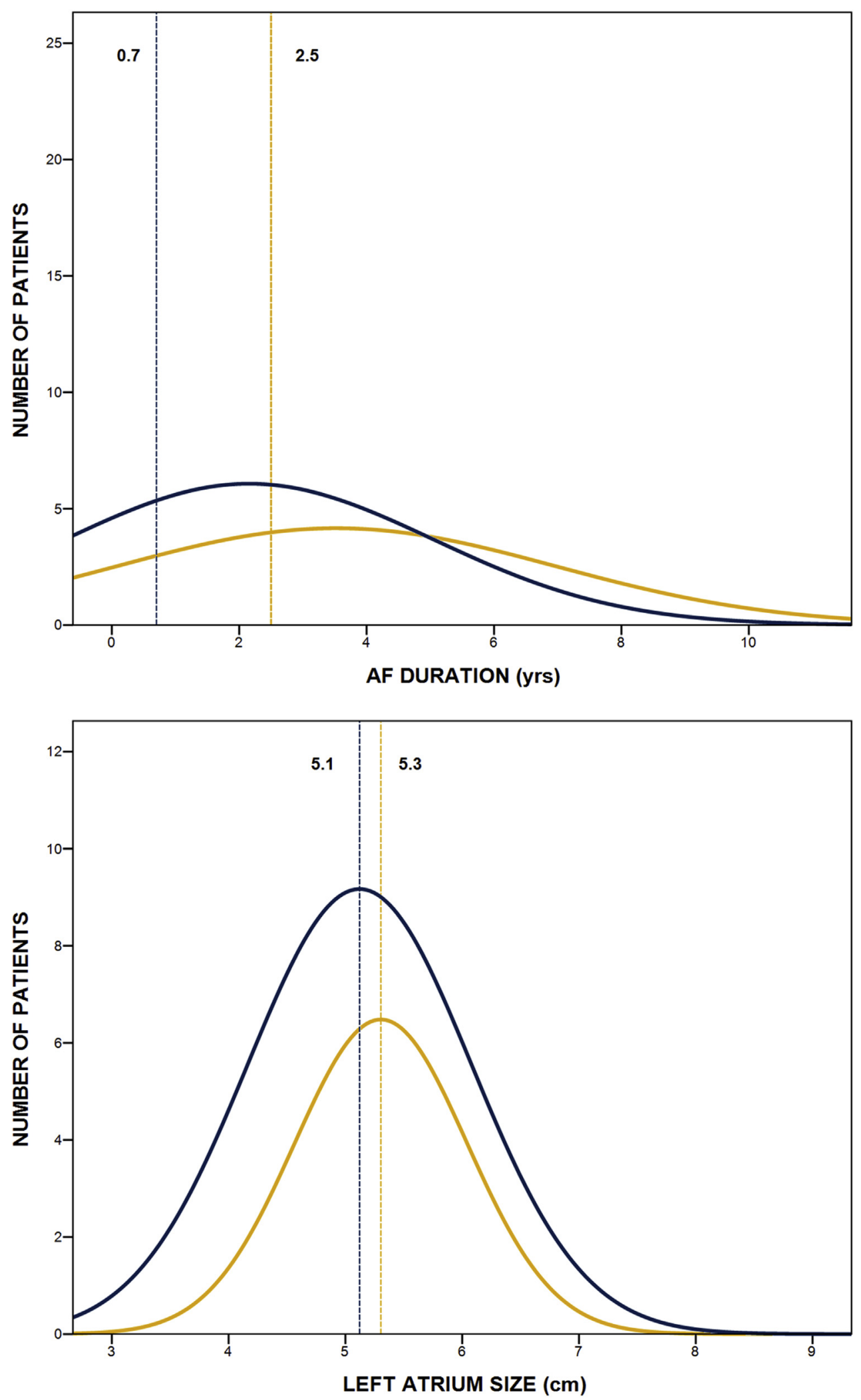

FIGURE 3. Distribution lines from histograms of preoperative atrial fibrillation $(A F)$ duration and left atrium size for patients in sinus rhythm off antiarrhythmic drugs (blue line) and not in sinus rhythm off antiarrhythmic drugs (gold line) at 7 years. Vertical lines represent median values for AF duration and mean values for left atrium size by group. 


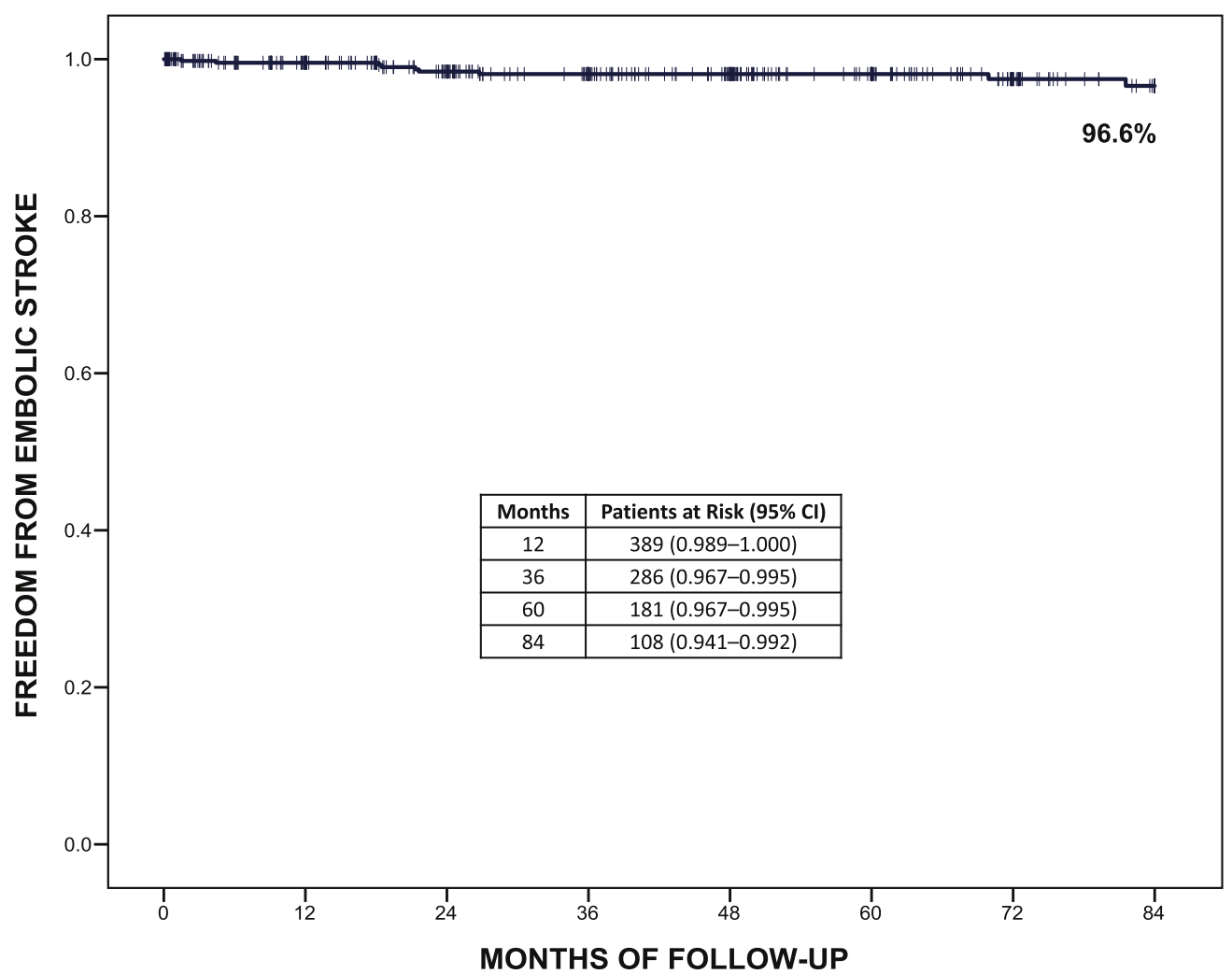

FIGURE 4. Cumulative freedom from embolic stroke during 7 years of follow-up. $95 \% C I, 95 \%$ Confidence interval.

the Kaplan-Meier analysis was $77 \%$ (95\% confidence interval, 0.717-0.823) at 7 years.

\section{DISCUSSION}

The results of this study demonstrate that patients undergoing mitral valve surgery and CM III/IV procedures should expect good early and late outcome. The surgical procedures were performed by multiple surgeons achieving acceptable rates of sinus rhythm at 7 years with a notably low stroke rate. The factors associated with atrial arrhythmia recurrence during 7 years were longer preoperative duration of $\mathrm{AF}$, combination ablative energy source (bipolar radiofrequency and cryothermal energy), and lower surgeon experience with surgical ablation.

Since 1987, the CM procedure transitioned from the cutand-sew technique to a device-based surgical ablation for $\mathrm{AF}$. The only 2 devices that were identified as reliable and consistent for transmural lesions in cases performed with cardiopulmonary support are the bipolar radiofrequency clamp and the cryothermal probes. ${ }^{6}$ Therefore, this series is unique not only in size and duration of follow-up but also due to the consistent use of these 2 energy sources recognized for consistent transmurality, either as a combination (bipolar radiofrequency and cryothermal) or as a standalone (cryothermal) to perform the CM III/IV procedure. ${ }^{6}$

The decision making surrounding the inclusion of surgical ablation in the concomitant setting when indicated is still evolving. Currently there is clear variability among surgeons on the degree and type of surgical ablation procedures performed. ${ }^{4,15}$ The decision is not only dependent on the pathophysiology of AF, but often also related to the unsupported concern of increased risk for morbidity and mortality associated with concomitant surgical ablation. Previous reports by us and by others demonstrated that surgical ablation in the concomitant setting was not associated with increased operative risk. ${ }^{16,17}$ More importantly recent guidelines clearly documented and recommended addition of surgical ablation to improve perioperative morbidity and mortality. ${ }^{5,6}$ Another recognized challenge is the inconsistency associated with the type of lesion set and energy sources. The Society of Thoracic Surgeons guidelines clearly state that mitral valve patients with more advanced atrial fibrillation and increased left atrium size should have a biatrial surgical ablation to include the $\mathrm{CM}$ procedure because it is associated with a superior outcome. ${ }^{5}$ The expectations are that these new guidelines and ongoing educational efforts 
will improve the ability of surgeons to make the right decisions regarding surgical ablation.

In the present study, perioperative mortality and morbidity were acceptable and a low observed-toexpected ratio of 0.55 for mortality was achieved. The safety of the procedure and the potential early influence on outcomes should be considered when operating on patients with coronary artery disease or structural heart disease with AF. The evidence and the recognition of the importance of AF should support the decision-making process to perform surgical ablation for AF when indicated. Long-term follow-up for such patients should be focused on the success in ablating AF, thromboembolic rate, anticoagulation, and survival. The success rate in our series diminished over the 7 years of follow-up and the most important factors associated with recurrence of atrial arrhythmia were longer duration of AF, ablation using a combination of bipolar radiofrequency and cryothermal energy devices rather than cryothermal probes alone, and lower surgeon experience. Our group has observed potential differences in success associated with cryothermal only CM procedures. It is unclear what the mechanism is to explain this difference in outcome, but it is a consistent finding in a few of our recent studies and observations. ${ }^{18}$ One potential explanation is that the cryothermal devices are not as userdependent compared with the bipolar radiofrequency devices. There is also a need to improve our understanding of the potential influence of atrial tissue remodeling on the radiofrequency ablation algorithm. It would be very important to design an appropriately powered prospective study to clarify this important result.

In the present study, the distribution of AF duration was shown to be shifted toward higher values in patients with atrial arrhythmia recurrence at 7 years after surgery, whereas the distributions for left atrium size were comparable for patients with and without recurrences at 7 years. This study is not the first to suggest that preoperative AF duration is likely the most important variable directly related to $\mathrm{AF}$ that is associated with long-term success of the CM procedure. The group from Washington University reported on their 5-year results with the $\mathrm{CM}$ procedure and found that longer duration of AF was significantly associated with failure. ${ }^{19}$ The duration of AF before surgery may have a direct influence on the degree of atrial tissue remodeling and complexity of the atrial substrate. Therefore, the decision to modify the CM procedure to a simpler left atrial lesion set based on the type of AF may be misleading because patients with long duration of paroxysmal AF may have more advanced disease than realized, especially if there are signs of left atrium enlargement. ${ }^{20,21}$ Our experience suggests that follow-up should be modified in such patients. Currently, the clinical focus of the referring cardiologist during follow-up is on the non-AF component of cardiovascular disease with no real involvement of the surgeons and electrophysiologists if and when AF recurs. A team approach to these patients could help improve long-term results because AADs and follow-up catheter ablations can improve our understanding of long-term recurrence and patient outcomes. ${ }^{22}$ Because all patients in this study had mitral valve surgery as well, it would be important to include the status of the mitral valve surgery at the time of failure because it may have a direct influence on left atrial pressure and size. To date there is no information about the potential influence of deteriorated mitral valve surgery and AF recurrence rate, especially when the follow-up period is long enough to detect such changes.

The recently published American Association for Thoracic Surgery guidelines state for the first time the importance of training and guidance to improve surgical treatment of $\mathrm{AF}^{6}{ }^{6}$ In the present study, as in others before, surgeon experience at the time of the procedure was found to be an important factor for long-term success. The CM procedure is composed of multiple steps that each must be performed accurately and precisely. It is also imperative for surgeons to be fully familiar with the nature of different ablation devices as well as all technical aspects related to the application of each lesion to avoid gaps, incomplete ablation lines, and misplaced lesions that may influence patient outcomes. To that end, The American Association for Thoracic Surgery guidelines also highly recommend personal proctorship to surgeons following training when performing their first 3 to 5 surgical ablation cases.

The present study provides unique information on thromboembolic strokes, bleeding complication and anticoagulation. During 7 years of follow-up, freedom from stroke was $96.6 \%$ (0.4 strokes per 100 patient-years). Recently, the stroke rate following catheter ablation was demonstrated to be lower than patients in AF, but higher than reported in the present study and with significantly higher rates of treatment with oral anticoagulation medication and bleeding complications. ${ }^{23}$ The present results are important and demonstrate significant reduction in the risk of thromboembolic and bleeding events. Stroke reduction over time was reported in the past and likely represents a combination of relatively good success rate, lower atrial arrhythmia burden, and management of the left atrial appendage. ${ }^{7,24}$ When reporting on atrial arrhythmia according to the HRS guidelines, any event longer than 30 seconds is considered a failure to achieve the rhythm goal. ${ }^{9}$ In our series only $22 \%$ had continuous atrial arrhythmia documented at 7 years. Although the long-term monitoring used in the majority of patients in this study was 24-hour Holter, it is reasonable to assume lower atrial arrhythmia burden in the majority of patients. The other important aspect of the procedure is management of the left atrial appendage with the potential influence on the low rate of thromboembolic events even in patients who experienced a recurrence. The relative contribution of each aspect of the procedure on the very low stroke 
rate and the success in reducing the number of patients on anticoagulation and bleeding complications at follow-up is yet to be determined. ${ }^{25}$

It is important to recognize that the electrophysiological mechanism of the CM procedure failure was not evaluated in the vast majority of patients in this series and this clinical phenomenon is very common in global practice. Future studies should focus on protocols that will include such an approach as well as a careful assessment of the status of the mitral valve and other valves, if treated in the original surgery, at the time of failure. Such an approach will provide important information regarding the potential association between late hemodynamic changes and recurrence of atrial arrhythmia following surgical ablation.

\section{Limitations}

A limitation of this study is that because rhythm status was not continuously monitored, it is possible that patients who are in sinus rhythm at scheduled time points may have experienced atrial arrhythmia recurrences in between, which were not captured. However, an ideal monitoring strategy for patients after surgical ablation for AF has not yet been determined. Implantable devices that continuously monitor have been shown to have high false positives and demonstrated to have no advantage over HRS guidelines for follow-up. ${ }^{26}$ The Reveal LINQ device (Medtronic) has been shown to have limitations in patients with low AF burden, which is common for patients following the CM procedure, and is also limited by battery life ( 3 years) and cost. $^{27}$

It is also possible that the results from this study with highly experienced surgeons may not be generalizable to other centers. Further emphasis on training and increasing mentored surgeon experience can improve the success of $\mathrm{CM}$ procedures in combination with mitral valve surgery. Lastly, although descriptive statistics comprised the majority of study analyses, the comparative statistics conducted may have been limited in power by low event rates and further studies are necessary to replicate the results from these analyses.

\section{CONCLUSIONS}

In this large cohort study, we reported on 7-year outcomes for patients who underwent concomitant CM III/IV procedures and mitral valve surgery. The safety of this approach is encouraging and replicates findings reported by our group and others. The long-term success rate is acceptable with important influence on outcome associated with the type of energy source, surgeon experience, and training. A very low stroke rate of 0.4 strokes per 100 patient-years of follow-up was documented with the majority of patients not on oral anticoagulation medication. These findings and the supporting data from recent guidelines should be considered by surgeons when operating on patients with significant history of AF.

\section{Webcast}

You can watch a Webcast of this AATS meeting presentation by going to: https://aats.blob.core.windows.net/media/ 17AM/2017-05-02/BallroomABC/05-02-17_BallroomABC_ 0851_Ad.mp4.

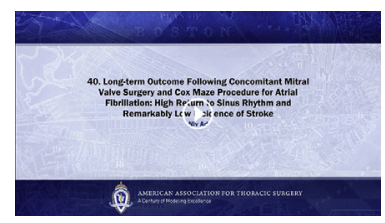

\section{Conflict of Interest Statement}

N.A. is a consultant for Medtronic, a member of the speaker's bureau for AtriCure, proctor and member of the speaker's bureau for LivaNova, on the advisory board for Nido Surgical, and co-owner of Left Atrial Appendage Occlusion, LLC. All other authors have nothing to disclose with regard to commercial support.

All surgical cases were performed at Inova Fairfax Hospital in Falls Church, Va. Data were collected and maintained by Deborah Lamont, BSN, RN, and Graciela Pritchard, BS, at Inova Fairfax Hospital in Falls Church, Va. The authors thank their colleagues for their contributions.

\section{References}

1. Kannel WB, Abbott RD, Savage DD, McNamara PM. Epidemiologic features of chronic atrial fibrillation: the Framingham study. N Engl J Med. 1982;306: 1018-22.

2. Dorian P, Jung W, Newman D, Paquette M, Wood K, Ayers GM, et al. The impairment of health-related quality of life in patients with intermittent atrial fibrillation: implications for the assessment of investigational therapy. J Am Coll Cardiol. 2000;36:1303-9.

3. Chatap G, Giraud K, Vincent JP. Atrial fibrillation in the elderly: Facts and management. Drugs Aging. 2002;19:819-46.

4. Ad N, Suri RM, Gammie JS, Sheng S, O’Brien SM, Henry L. Surgical ablation of atrial fibrillation trends and outcomes in North America. J Thorac Cardiovasc Surg. 2012;144:1051-60.

5. Badhwar V, Rankin JS, Damiano RJ, Gillinov AM, Bakaeen FG, Edgerton JR, et al. The Society of Thoracic Surgeons 2017 clinical practice guidelines for the surgical treatment of atrial fibrillation. Ann Thorac Surg. 2017;103:329-41.

6. Ad N, Damiano RJ Jr, Badhwar V, Calkins H, La Meir M, Nitta T, et al. Expert consensus guidelines: examining surgical ablation for atrial fibrillation. J Thorac Cardiovasc Surg. 2017;153:1330-54.

7. Bando K, Kasegawa H, Okada Y, Kobayashi J, Kada A, Shimokawa T, et al. Impact of preoperative and postoperative atrial fibrillation on outcome after mitral valvuloplasty for nonischemic mitral regurgitation. J Thorac Cardiovasc Surg. 2005;129:1032-40.

8. Lee R, McCarthy PM, Wang EC, Vaduganathan M, Kruse J, Malaisrie SC, et al. Midterm survival in patients treated for atrial fibrillation: a propensity-matched comparison to patients without a history of atrial fibrillation. J Thorac Cardiovasc Surg. 2012;143:1.

9. Calkins H, Kuck KH, Cappato R, Brugada J, Camm AJ, Chen SA, et al. 2012 HRS/EHRA/ECAS expert consensus statement on catheter and surgical ablation of atrial fibrillation: recommendations for patient selection, procedural techniques, patient management and follow-up, definitions, endpoints, and research trial design. J Interv Card Electrophysiol. 2012;33:171-257.

10. Cox JL, Schuessler RB, D’Agostino HJ, Stone CM, Chang BC, Cain ME, et al The surgical treatment of atrial fibrillation. III. Development of a definitive surgical procedure. J Thorac Cardiovasc Surg. 1991;101:569-83. 
11. Cox JL. The surgical treatment of atrial fibrillation. IV. Surgical technique. $J$ Thorac Cardiovasc Surg. 1991;101:584-92.

12. Damiano RJ, Bailey M. The Cox-maze IV procedure for lone atrial fibrillation. Multimed Man Cardiothorac Surg. 2007;2007:mmcts.2007.002758.

13. Ad N, Henry L, Hunt S, Stone L. The implementation of a comprehensive clinical protocol improves long-term success after surgical treatment of atrial fibrillation. J Thorac Cardiovasc Surg. 2010;139:1146-52.

14. Ad N, Holmes SD, Shuman DJ, Pritchard G, Miller CE. Amiodarone after surgical ablation for atrial fibrillation: is it really necessary? A prospective randomized controlled trial. J Thorac Cardiovasc Surg. 2016;151:798-803.

15. Ad N, Henry L, Hunt S, Holmes SD. Impact of clinical presentation and surgeon experience on the decision to perform surgical ablation. Ann Thorac Surg. 2013; 96:9.

16. Ad N, Henry LL, Holmes SD, Hunt SL. The impact of surgical ablation for atrial fibrillation in high-risk patients. Ann Thorac Surg. 2012;93:4.

17. Ad N, Holmes SD, Pritchard G, Shuman DJ. Association of operative risk with the outcome of concomitant Cox maze procedure: a comparison of results across risk groups. J Thorac Cardiovasc Surg. 2014;148:3027-33.

18. Ad N, Holmes SD, Rongione AJ, Massimiano PS, Fornaresio LM. Does surgical ablation energy source affect long-term success of the concomitant Cox maze procedure? Ann Thorac Surg. 2017;104:29-35.

19. Henn MC, Lancaster TS, Miller JR, Sinn LA, Schuessler RB, Moon MR, et al. Late outcomes after the Cox maze IV procedure for atrial fibrillation. $J$ Thorac Cardiovasc Surg. 2015;150:2

20. Lim HS, Hocini M, Dubois R, Denis A, Derval N, Zellerhoff S, et al. Complexity and distribution of drivers in relation to duration of persistent atrial fibrillation. J Am Coll Cardiol. 2017;69:1257-69.

21. Ad N, Holmes SD, Lamont D, Shuman DJ. Left-sided surgical ablation for patients with atrial fibrillation who are undergoing concomitant cardiac surgical procedures. Ann Thorac Surg. 2017;103:58-65.

22. Henry L, Durrani S, Hunt S, Friehling T, Tran H, Wish M, et al. Percutaneous catheter ablation treatment of recurring atrial arrhythmias after surgical ablation. Ann Thorac Surg. 2010;89:2.

23. Riley MP, Zado E, Hutchinson MD, Lin D, Bala R, Garcia FC, et al. Risk of stroke or transient ischemic attack after atrial fibrillation ablation with oral anticoagulant use guided by ECG monitoring and pulse assessment. J Cardiovasc Electrophysiol. 2014;25:591-6.

24. Cox JL, Ad N, Palazzo T. Impact of the maze procedure on the stroke rate in patients with atrial fibrillation. J Thorac Cardiovasc Surg. 1999;118:833-40.

25. Cox JL. Mechanical closure of the left atrial appendage: is it time to be more aggressive? J Thorac Cardiovasc Surg. 2013;146:1027.e2.

26. Damiano RJ, Lawrance CP, Saint LL, Henn MC, Sinn LA, Kruse J, et al. Detection of atrial fibrillation after surgical ablation: conventional versus continuous monitoring. Ann Thorac Surg. 2016;101:8.

27. Mittal S, Rogers J, Sarkar S, Koehler J, Warman EN, Tomson TT, et al. Realworld performance of an enhanced atrial fibrillation detection algorithm in an insertable cardiac monitor. Heart Rhythm. 2016;13:1624-30.

Key Words: cardiac surgery, atrial fibrillation, mitral valve

\section{Discussion}

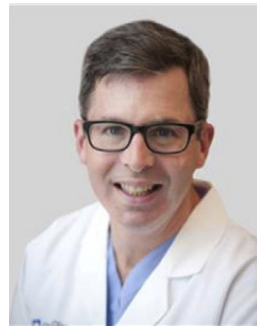

Dr Mark Gillinov (Cleveland, Ohio). That was great and you continue to lead in the field of surgical ablation. By way of disclosure, I have served as a consultant to companies in the ablation field but none of that activity will be relevant to the discussion.

I want to point out a couple of strengths before asking you questions. You said your results are acceptable. I would say they are spectacular, and you truly set the bar for the rest of us. You report on 473 patients, most of whom had nonparoxysmal atrial fibrillation (AF). So these are tougher patients. And, as I said, you got a very high return to sinus rhythm and an extraordinarily low risk of late stroke.

I want to touch on a couple of things. Three questions. First, you have said this before and you highlight it today: Surgeon experience counts. Many of us view the maze procedure or an ablation procedure - you did a full maze - as an add-on to the main operation of the mitral valve. What does the experienced surgeon do differently from the other surgeon that makes a difference?

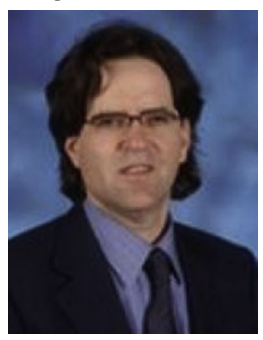

Dr Niv Ad (Falls church, VA). Thank you for your kind comments. It is an excellent question. Surgical ablation is really unique compared with what we do every day; it is a collision between technique and technology. And I think that this is what we don't really get most of the time. It is really important for every surgeon to understand the technology that is being used, what it is meant to do and what it isn't meant to do, and also how to apply those lesions. The experience basically goes into those two aspects and who trained you.

Now, we don't have an official training program on AF and there are not many surgeons who can really train you on the full lesion set and so on and so forth. For me, it was a special experience coming from a different country trying to teach surgeons who have had 15 to 20 years longer experience compared with me and teaching them how to do it. But I think it is important for us to show how to apply a lesion with a cryo, how to use the bipolar and so on and so forth.

I think you get to some kind of an acceptable understanding by about 50 cases, but I don't have any hard evidence to support this number.

Dr Gillinov. A second question: All of the patients in this series had a biatrial lesion set, a Cox maze III or IV. Is that the lesion set that you recommend in everyone with mitral valve disease?

Dr Ad. Yes, I think so, but highly regarded surgeons like Pat McCarthy, who is not here, may not agree. AF is a moving target; the mechanism you ablate today may not be the reason for recurrence tomorrow. You have to have a lesion set that can address most of it at the time of surgery when you move forward.

We did look at a subgroup of patients with left atria alone, and what we found was very interesting. If you take those risk factors, the full risk factors that I just mentioned, if you have zero risk factors, meaning small atrium, short duration, young age, and so on and so forth, you can have a reasonable success rate, actually an excellent success rate: at 2 years with about $90 \%$. We published that. But when you have 1 or 2 risk factors, which is changing the type of AF, increased age, you go down to about a $70 \%$ success rate at 1 year. So I think it is really important to choose the patients. 


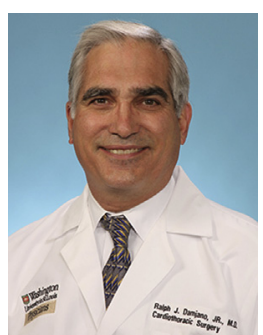

Dr Ralph Damiano (St Louis, Mo). Congratulations on both a wonderful study and your long-term follow-up. That is something that has been lacking in the literature and also something that requires a tremendous amount of effort. We can learn a lot from your data. This also is among the largest series of mitral patients presented.

My first question is that it seems that there was a steady recurrence rate over time, and we noticed the same when we reported our 5-year data. Could you elucidate the patients who did recur, was the recurrence mode AF or flutter? Can you give us some idea of the burden of $\mathrm{AF}$ in those recurrent patients in a way that may help us improve our results?

My second part of that question is, I assume your study included patients with degenerative, rheumatic and functional mitral regurgitation (MR). I completely agree with you, we don't know much about the mechanisms of AF in individual patients. I imagine it may be different depending on what was the actual etiology of the MR. Did the type of mitral regurgitation have any influence on late recurrence? We know for functional MR, for instance, we are not as good and we have a lot of recurrent MR. Did you look at your AF recurrences? Did they have, at the time of recurrent $\mathrm{AF}$, recurrent valvular disease, or was this unrelated?

Dr Ad. Those are all very good questions and your remarks are very kind to us and the work we have done. We don't know about the mitral valve disease, and this is why I mentioned in my presentation that it is really important to start to look into it, because I totally believe that longterm follow-up should take into account the stability of the valve repair or replacement, it is not electrophysiology only. As for duration of atrial fibrillation, as you pointed out, it is probably the most important factor, duration is associated with remodeling, and I think the longer duration you have, recurrence of structural heart disease and failure will have more impact. So shorter duration of atrial fibrillation on the other hand, may be less impacted by recurrence of mitral valve disease; longer duration is going to be important for recurrence. So it is showing us that remodeling is not fully reversible, even after 7 years. We don't have the data and we don't know.

I had a slide regarding AF burden, but I took it out because of the 6 minutes. But you are completely right; not all those that failed were with continuous AF. Actually most of them were with AF between 5 minutes and 1 hour, which is a lower burden. So I think that is also very, very important. That steady decrease over time needs to be studied more thoroughly, and I think we need to move forward with more collaboration with our colleagues, electrophysiologists, and study those failures routinely. As you alluded to, most of the patients do not recur with AF, they recur either with some kind of a flutter that is not like the classical because it is some macroreentrant circuit somewhere or focal arrhythmia. So I think this needs to be studied more and we have way more work to do just to clarify all those points.

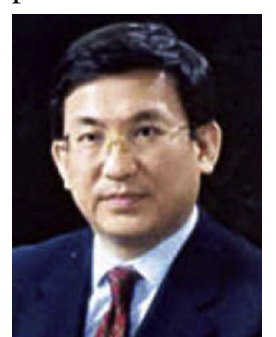

Dr Ko Bando (Tokyo, Japan). We continue to learn a lot from you. I have a question regarding your left atrial appendage closure technique. Your results that you showed were such excellent results with a very low stroke rate. On the other hand, Marc, you published back in 2008, and the success rate of left atrial closure is about $40 \%$. It depends on the excision or exclusion. Dr Ad, what is your technique of left atrial appendage closure? How can you assure your left atrial appendage closure is complete?

Dr Ad. That is an excellent question. I wish I had the numbers. That is why I don't want to make it up. But the majority of the patients with an open sternotomy mitral got their appendage excised by very experienced surgeons and the excision is reasonably good; we saw it by echocardiogram. We have closure from the inside, and we reported the closure from the inside 2 layers, although in the operating room looked very, very good, at about 6 months you have recanalization to some extent, about $5 \%$. So we moved from that almost completely unless it is a redo and you don't want to do so much work outside. For the minis we do close the appendage now through the transverse sinus with a clip, and we use more and more clips in the open technique as well.

I don't have the precise numbers, but given the fact that the stroke rate is so low, I think we can assume we did a good job with closing the left atrial appendage.

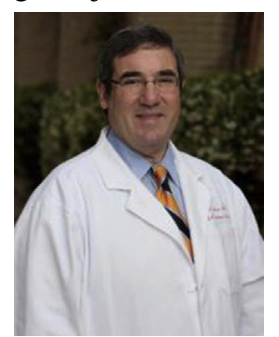

Dr Richard J. Shemin (Los Angeles, Calif). I think the results that you have shown us today are excellent, and the main point, to me, is that you need a complete lesion set and you need to be experienced to get it right the first time, and I think we are all concerned that over time there is deterioration in the outcomes and the results. You have mentioned that maybe it is the recurrence or what is happening to the mitral valve that may be part of that equation.

But I want to focus on the postoperative care and longitudinal follow-up. How important to achieve these results is actually having an AF heart team partnering with an electrophysiologist, making sure that surgeons will be comfortable in the follow-up of the valve or the valve repair? Very often we ignore the electrophysiologic components, the management of the drug therapy, the desire to get off antiarrhythmic drugs to improve outcomes. So if you could just focus on putting together the right team to maximize the achievement of long-term results. 
Dr Ad. We are over the time, so we don't have time to really answer, but you are right, dead on, on all points. Let me just give you one number. I think this is important for everyone.

We decided to start this follow-up program when we realized that after six months, only $13 \%$ of our patients were cardioverted for recurrence, and when I went to my colleagues, the electrophysiologists, I asked them how many patients that they ablated and failed during the first 6 months are being cardioverted? They said $100 \%$. This was 11 years ago, and it gave me the idea of how instrumental close followup can be for patients. So we need to follow those patients, indefinitely.

Dr Gillinov. One last question: As you follow patients, how hard do you try to get them back into sinus rhythm when the AF recurs? Let's say you are a year or 2 after the maze procedure, everything has looked good, and now they are back in AF. What do you do?

Dr Ad. You need to start from the basics. It is almost like you have recurrent coronary artery disease. So you go back, you put them on antiarrhythmic drugs, you cardiovert them, and if it doesn't work, then you try another drug and then ablation, if needed. But you need to try hard, yes.

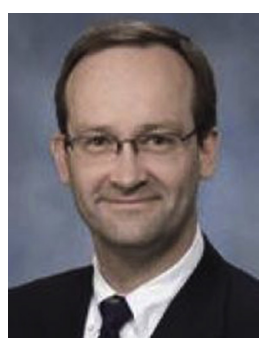

Dr Thoralf Sundt (Boston, Mass). My question relates to why we are doing the operation at all. You will see in the newest guidelines update that there is a statement that you should not do a maze procedure associated with a mitral valve procedure for the purpose of getting a patient off anticoagulation therapy. And that is because in the discussion, the debate, the back and forth in the guidelines committee, the fact is that the cardiologists want to anticoagulate them anyway if the patient has a CHADS 2 score $>2$. Period. Whether you have done a maze or not, even if you have been successful in converting them to sinus rhythm, it is really hard to get electrophysiologists to agree to take them off anticoagulation therapy. This was distressing to me because I always thought the home run was to do a mitral valve repair and a maze with conversion to sinus rhythm so that a patient would not need anticoagulation therapy. But you only have to be female greater than the age of 65 years and you have already got a CHADS score $>2$. Boom, you are on anticoagulant therapy.

So why are we doing the operation?

Dr Ad. Marc?

Dr Gillinov. Not because it is fun. We are doing this operation to improve quality of life, make people feel better, possibly to improve survival, and definitely to reduce risk of stroke. In addition, if I see continued sinus rhythm at 6 months and I know the appendage is occluded, I take the patient off anticoagulation.

Dr Ad. I will be very brief. I think you are right, but we do the operation because we know that when you have valvular heart disease and atrial fibrillation that is a predictor for stroke and death. So whether you stop anticoagulation or not or whether you are on any anticoagulation, if you are in $\mathrm{AF}$, you have a high risk of stroke. That is a very important point, but it may be irrelevant in the decision, because we reduce stroke regardless of anticoagulation.

Our research shows that CHADS score is probably irrelevant for surgical patients. We showed it in 2 very detailed articles that we published in the past. So what we do, first patients have to be in sinus rhythm and then the appendage needs to be shown to be closed in 6 months by echocardiogram and no echogenic smoke documented. But we truly have a gap we need to work on with our colleagues to come up with our precise guidelines based on evidence that we currently don't have. It is an important gap. Based on my experience, it is important to offer patients sinus rhythm, even if anticoagulation is not discontinued. 